\title{
Fasting during the ramadan: a challenge for patients with adrenal insufficiency
}

\author{
Miguel Debono ${ }^{1}$
}

Received: 10 April 2017 / Accepted: 12 May 2017 / Published online: 20 May 2017

(C) Springer Science+Business Media New York 2017

During the Ramadan, Muslims fast every day, from dawn to sunset, for a whole month. The fast is a stressful period when individuals abstain from eating and drinking and are therefore at risk of dehydration, fainting, low glucose levels, intense thirst, and asthenia. In patients with adrenal insufficiency who are deficient in one of the major metabolic and stress hormones in the body, cortisol, this may be fatal. Daily treatment with glucocorticoids usually hydrocortisone is essential. In the paper by Chihaoui et al. [1], patients with adrenal insufficiency were mostly on twice daily hydrocortisone regimes with the higher dose during fasting administered at the sunset meal and the lower dose at the predawn meal. During Ramadan taking medicine orally is considered breaking the fast. For many fasting Muslims, strictly following their religious needs, changing the timing of their medications, in this case hydrocortisone, is inevitable.

The physiological cortisol circadian rhythm is well defined with circulating levels rising at 02:00 to 04:00 h, reaching a peak an hour after waking, and after that gradually declining to lowest levels at midnight [2]. The cortisol rhythm regulates other bodily rhythms and acts as a secondary messenger between the central clock and peripheral clocks in different tissues and cells [3]. An example of this interaction is the relationship between the cortisol rhythm and the insulin resistance rhythm [4]. The

Miguel Debono

m.debono@sheffield.ac.uk

1 Consultant in Endocrinology and Honorary Senior Lecturer, Department of Endocrinology, Royal Hallamshire Hospital, Sheffield Teaching Hospitals/University of Sheffield, Sheffield, UK cortisol rhythm directly influences insulin resistance. If cortisol levels are abnormally high during the quiescent phase of the rhythm, that is between 19:00 and 02:00 h, this predisposes to abnormal glucose levels, increasing the risk of developing insulin resistance [5]. Similarly when subjects sleep out of phase to their normal sleep cycle, such as shift workers or during jet lag, the cortisol rhythm is misaligned hence resulting in glucose intolerance [6]. Therefore taking a higher dose of conventional hydrocortisone, which peaks within 30 to $60 \mathrm{~min}$ in the evening, is not advisable as this may cause metabolic disturbances and sleep problems. This may also result in fatigue and tiredness during the day. Superimposed on the stress of the fast this puts patients with adrenal insufficiency under a high risk for an adrenal crisis.

In addition to this, low cortisol levels during the rest of the night have been associated with low concentrations of glucose, fatty acids, amino acids, and 3-hydroxybutyrate [7]. This could possibly explain why patients with adrenal insufficiency complain of fatigue early in the morning [8], and why patients on traditional regimes of conventional hydrocortisone that do not produce the early morning rise in cortisol are at risk of nocturnal hypoglycaemia [9]. In the presence of an early morning rise in cortisol these metabolites could all increase, potentially making one feel more energetic on waking [7]. To compensate for this, taking the higher dose of hydrocortisone in the morning at dawn slightly later than the early morning rise in cortisol could reduce late morning tiredness. Unfortunately as the half-life of hydrocortisone is around $90 \mathrm{~min}$, and its effect may last up to 4 to $6 \mathrm{~h}$, during most of the day patients will still have cortisol levels well below normal, putting these patients at risk for adrenal crisis. This may result in hospital admissions and possible death. 
An adrenal crisis is a life threatening emergency. Patients with adrenal insufficiency have 5-10 adrenal crises per 100 patient years. This is an important factor increasing mortality rate [10]. In the majority of cases, an adrenal crisis develops over hours, but sometimes may develop very rapidly making it hard to call for help in time. Rarely crises may be irreversible and treatment unsuccessful hence the importance of avoiding instances which increase the risk of this critical event [11]. Patient education is essential for crisis prevention. It is important that patients wanting to fast during the Ramadan are well educated, to the extent that they can lead their own management and guide people around them on how to cope with and prevent impending illness. To strengthen and support this point is that the only factor associated with the risk of complications during the study by Chihaoui et al. was knowledge about the disease. This is not straightforward as achieving maximum patient education is not simple and needs to be continuously reinforced. Patients should be provided with an emergency card, instructions on how to use stress doses of oral hydrocortisone and should be supplied with a parenteral hydrocortisone injection. Ideally patients or their close relatives should be taught how to use these injections. The injections provided should be practical and easy to use.

The development of new formulations of hydrocortisone, with modified release mechanisms, could potentially reduce the risk for complications and adrenal crises during the Ramadan by extending 24-h cortisol exposure and delivering the right amount of cortisol in the appropriate time of the day. Chronocort ${ }^{\circledR}$, administered twice daily with the higher dose at sunset and the lower dose at dawn has been shown to approximate the physiological cortisol circadian rhythm [12]. This formulation would allow those patients wanting to fast during the Ramadan respect their obligations and not break the fast. Other options would be the use of Plenadren $^{\circledR}$, a once daily immediate-sustained release formulation which can be administered at dawn and can help with compliance [13]. Subcutaneous infusions of hydrocortisone [14] providing circadian cortisol, do not entail any drinking but then are cumbersome. Patients require training to learn how to use the pumps. This strategy might be too complex to familiarise with rapidly within a month. If novel formulations are unavailable, one can consider the use of daily 4 or $5 \mathrm{mg}$ prednisolone administered at dawn [15]. To avoid metabolic complications and sleeping problems, it is not recommended to administer a dose of prednisolone at sunset. Dexamethasone, a long acting glucocorticoid, carries a high risk of side effects and ideally should not be used in this setting.

For patients with adrenal insufficiency fasting in the Ramadan is a challenge. A higher conventional hydrocortisone dose in the morning is more suitable than at sunset. Modified release formulations of hydrocortisone delivering more physiological cortisol exposure could potentially reduce complications. Most importantly patients need to be educated and have full knowledge of their disease. It is essential that this knowledge is continuously strengthened at each health worker to patient encounter. By doing this, the risks of the Ramadan fast for these patients can be significantly reduced allowing these individuals to fulfil their religious responsibilities during this intense period of stress.

\section{Compliance with ethical standards}

Conflict of interest The author declares that they have no competing interests.

Ethical approval This article does not contain any studies with human participants or animals performed by the author.

\section{References}

1. M. Chihaoui, F. Chaker, M. Yazidi et al., Ramadan fasting in patients with adrenal insufficiency. Endocrine 55, 289-295 (2017)

2. M. Debono, C. Ghobadi, A. Rostami-Hodjegan et al., Modifiedrelease hydrocortisone to provide circadian cortisol profiles. J. Clin. Endocrinol. Metab. 94, 1548-1554 (2009)

3. A.Y. So, T.U. Bernal, M.L. Pillsbury, K.R. Yamamoto, B.J. Feldman, Glucocorticoid regulation of the circadian clock modulates glucose homeostasis. Proc. Natl. Acad. Sci. USA 106, 17582-17587 (2009)

4. L. Plat, M.M. Byrne, J. Sturis et al., Effects of morning cortisol elevation on insulin secretion and glucose regulation in humans. Am. J. Physiol. 270, E36-E42 (1996)

5. L. Plat, R. Leproult, M. L'Hermite-Baleriaux et al., Metabolic effects of short-term elevations of plasma cortisol are more pronounced in the evening than in the morning. J. Clin. Endocrinol. Metab. 84, 3082-3092 (1999)

6. C.J. Morris, T.E. Purvis, J. Mistretta, F.A. Scheer, Effects of the internal circadian system and circadian misalignment on glucose tolerance in chronic shift workers. J. Clin. Endocrinol. Metab. 101, 1066-1074 (2016)

7. S. Dinneen, A. Alzaid, J. Miles, R. Rizza, Metabolic effects of the nocturnal rise in cortisol on carbohydrate metabolism in normal humans. J. Clin. Invest. 92, 2283-2290 (1993)

8. K.A. Al-Shoumer, K. Ali, V. Anyaoku, R. Niththyananthan, D.G. Johnston, Overnight metabolic fuel deficiency in patients treated conventionally for hypopituitarism. Clin. Endocrinol. 45, 171-178 (1996)

9. P. Cambiaso, R. Schiaffini, G. Pontrelli et al., Nocturnal hypoglycaemia in ACTH and GH deficient children: role of continuous glucose monitoring. Clin. Endocrinol. 79, 232-237 (2013)

10. S. Hahner, M. Loeffler, B. Bleicken et al., Epidemiology of adrenal crisis in chronic adrenal insufficiency: the need for new prevention strategies. Eur. J. Endocrinol. 162, 597-602 (2010)

11. B. Allolio, Extensive expertise in endocrinology. Adrenal crisis. Eur. J. Endocrinol. 172, R115-R124 (2015)

12. M. Whitaker, M. Debono, H. Huatan, D. Merke, W. Arlt, R.J. Ross, An oral multi-particulate, modified release, hydrocortisone replacement therapy that provides physiological cortisol exposure. Clin. Endocrinol. 80, 554-561 (2013) 
13. G. Johannsson, A.G. Nilsson, R. Bergthorsdottir et al., Improved cortisol exposure-time profile and outcome in patients with adrenal insufficiency: a prospective randomized trial of a novel hydrocortisone dual-release formulation. J. Clin. Endocrinol. Metab. 97, 473-481 (2012)

14. M. Oksnes, S. Bjornsdottir, M. Isaksson et al., Continuous subcutaneous hydrocortisone infusion versus oral hydrocortisone replacement for treatment of Addison's disease: a randomized clinical trial. J. Clin. Endocrinol. Metab. 99, 1665-1674 (2014)

15. E.S. Husebye, B. Allolio, W. Arlt et al., Consensus statement on the diagnosis, treatment and follow-up of patients with primary adrenal insufficiency. J. Intern. Med. 275, 104-115 (2014) 